\title{
Itineraires Therapeutiques Des Malades De L'ulcere De Buruli Et Difficultes De Prise En Charge Hospitaliere A Djekanou (Cote d'Ivoire)
}

\author{
Adjet A. Abel, Enseignant - Chercheur \\ Université Jean Lorougnon GUEDE, \\ Centre Suisse de Recherches Scientifiques en Côte d'Ivoire
}

doi: 10.19044/esj.2016.v13n3p197 URL:http://dx.doi.org/10.19044/esj.2016.v13n3p197

\begin{abstract}
Among the neglected tropical disease, Buruli ulcer (BU) is a real public health problem in Côte d'Ivoire. Facing this pathology, international and national institutions to combat advocate for people in endemic areas early detection and immediate use of support centers at the first signs. However, patients continue to attend late care centers with large ulcerative lesions. Therefore, healing requires a long-term hospitalization that requires medical and surgical treatment. The main objective of this study is to analyze the factors that change the therapeutic route of BU patients and constraints socio - economic issues related to their hospitalisation. This is a qualitative and quantitative study. We used the technique of reasoned choice. It covered 55 patients met at the Djekanou care center in the Toumodi Health District. Data were collected through structured and semi - structured interviews based on questionnaires, individual interview guides and focus groups.

A significant proportion of patients with BU are children and adolescents. The sick are accompanied by a parent who takes care of them. Awareness campaigns, community health workers, television and radio did not really help to change the therapeutic itinerary of patients. The patients encountered testify to the ineffectiveness of traditional care. The hospitalization of patients is marked by difficulties of several kinds.
\end{abstract}

Keywords: Buruli ulcer, Care, Constraints, Hospitalization, Côte d’Ivoire

\section{Resume}

Parmi les maladies tropicales négligées, l'ulcère de Buruli (UB) constitue un véritable problème de santé publique en Côte d'Ivoire. Face à cette pathologie, les institutions internationales et nationales de lutte préconisent aux populations des zones endémiques un dépistage précoce et un recours immédiat aux centres de prise en charge dès les premiers signes. 
Cependant, les malades continuent à fréquenter tardivement les centres de prise en charge avec de larges lésions ulcératives. La plupart d' entre eux recourent aux tradithérapeutes avant de fréquenter les centres de prise en charge. Dès lors, leur guérison passe par une hospitalisation de longue durée qui nécessite un traitement médical et chirurgical. L’objectif principal de cette étude est d'identifier les canaux d'information qui conduisent les malades au centre de prise en charge après un détour à la médecine africaine et analyser les contraintes sociales et économiques liées à l'hospitalisation de ceux - ci. C'est une étude qualitative et quantitative. Nous avons utilisé la technique du choix raisonné. Elle a porté sur 55 malades rencontrés au centre de prise en charge de Djekanou dans le District sanitaire de Toumodi. Les données ont été collectées par des entretiens structurés et semi - structurés à partir de questionnaires, guides d'entretien individuels et focus groups.

Une proportion importante des malades atteints de l'UB sont des enfants et des adolescents. Les malades sont accompagnés d'un parent qui s'occupe d'eux. Les campagnes de sensibilisation, les Agents de Santé Communautaires, la télévision et la radio n’ont pas véritablement contribué à changer l'itinéraire thérapeutique des malades. Les malades rencontrés témoignent de l'inefficacité des soins traditionnels. L'aggravation de la maladie les a conduit au centre de soin. L'hospitalisation des malades est marquée de difficultés de plusieurs ordres.

Mots-clés: Ulcère de Buruli, Soins, Contraintes, Hospitalisation, Côte d'Ivoire

\section{Introduction}

L’UB fait partie des maladies tropicales négligées. Il touche en priorité les populations rurales de nombreux pays d'Afrique subtropicale (Aujoulat et al; 1996). En Côte d'Ivoire, le premier cas a été observé en 1978 chez un enfant de nationalité française ayant fréquenté les bords du lac Kossou. Mais ce n'est qu'en 1989 que cette maladie se révèle à la population ivoirienne avec l'apparition du foyer endémique dans la région de Daloa. Elle fut caractérisée de «maladie mystérieuse de Daloa». Depuis lors, l'UB connaît une ampleur inquiétante liée à son évolution numérique et spatiale.

Plusieurs districts sanitaires sont touchés. Plus de 2000 nouveaux cas sont dépistés chaque année. La Côte d'Ivoire est le pays le plus touché au monde (PNLUB, 2009). C'est un épineux problème de santé publique, un handicap au développement et une menace émergente au plan socio démographique, socio - économique, psychosocial au regard des conséquences que la maladie engendre. Ce sont entre autres, les séquelles invalidantes, les déformations de membres, des handicaps... 
Du fait des larges lésions ulcératives qu'occasionne cette maladie et conscient des souffrances liées à celle - ci, le PNLUB conseille aux malades et aux populations des zones endémiques un dépistage précoce et un recours immédiat aux centres de prise en charge. Il s’attèle à apporter une réponse adéquate à cette menace «sérieuse» du capital humain que constitue cette maladie. De ce fait, des activités de sensibilisations ont été organisées. Des relais communautaires et des Agents de Santé Communautaires (ASC) ont été formés dans différents districts sanitaires. Des ateliers de formations et de renforcement de capacités se sont adressés aux prestataires des centres spécialisés. Plus de 300 centres de santé dans différents districts sanitaires ont été érigés en centre de prise en charge de premiers niveaux et de soins. Les soins médicaux et chirurgicaux sont offerts gratuitement aux malades (PNLUB, 2009).

Malgré toutes ces actions, des malades continuent de recourir aux thérapeutes traditionnels et fréquentent tardivement les centres de soins avec de larges ulcérations. Pourtant les prises en charge médicale et chirurgicale sont gratuites. Cette réalité n'est pas spécifique à la Côte d'Ivoire mais celle de nombreux pays africains.

L'OMS (2013) soutient ainsi que les consultations au niveau des centres médicaux ont lieu tardivement, en phase d'ulcération pour $84 \%$ des patients. Le recours tardif des malades aux centres de prise en charge et la prédominance de la médecine africaine dans leur itinéraire thérapeutique ont été abordés par plusieurs auteurs dans le cas d'observation liée à plusieurs pathologies.

Pour Meg (1992) (Cité par Sene en 2013), en raison des causes invisibles ou magico-religieuses que l'on attribue souvent à la maladie, nombreuses sont les personnes qui préfèrent s'en remettre au savoir empirique et à l'approche holistique du guérisseur traditionnel, plutôt que de recourir à la médecine moderne, également nommée " la médecine des blancs ». Ainsi, les guérisseurs traditionnels occupent donc une place très importante dans les sociétés traditionnelles africaines et leurs systèmes sanitaires.

Particulièrement, dans le cas de la lutte contre l’UB, plusieurs facteurs expliquent le phénomène.

Selon Kibadi (2004), les consultations au centre de prise en charge ont lieu parfois plus de 6 mois après l'apparition des premiers symptômes. Les raisons principales évoquées sont les variables économiques et l'accessibilité aux soins ainsi que les représentations de la maladie.

Pour Guedenon et al (1995); Sagno et al (2001); et Ymkje (2001) (Cités par Kibadi Kapy (2004) et Kibadi K. lui-même, le mauvais sort à l'origine de l'UB serait occasionné par l'action d'un objet maléfique déposé sur le chemin de la victime. Sagno et al. (Cités par Kibadi Kapya, 2004) ont 
également mis en évidence suite à une étude menée en Guinée que l’UB est principalement attribuée à des causes mystiques. Stienstra et al. (Cités par Kibadi et al, 2007) soulignent qu'au Ghana, 59\% des patients atteints d'UB considèrent avoir été victimes de pratiques de sorcellerie ou d'un mauvais sort.

En Côte d’Ivoire, en 2010, au plan national, sur 1659 malades, 972 soit 58,58 \% de malades ont eu recours à la médecine africaine avant les centres de prise en charge. Parmi Ceux-ci, 1105 malades soit 66,60\% présentaient des ulcérations dont 398 malades avec des limitations articulaires (PNLUB, 2010).

Pour Adjet (2016), dans le district sanitaire de Yamoussoukro, la proximité culturelle et géographique de la médecine africaine, les croyances culturelles et représentations de l'UB, les contraintes liées à l’hospitalisation et la fréquentation des centres justifient le recours tardif aux centres de soins.

Par ailleurs, ces détours à la médecine africaine occasionnent des complications, une hospitalisation de longue durée pour des populations des zones rurales souvent démunies. Cependant, les canaux d'information et les facteurs sociaux qui conduisent les malades à abandonner les soins traditionnels pour le centre de prise en charge sont peu documentés dans la littérature existante. De ce fait, très peu de données existent sur l'impact des activités de sensibilisation et d'information et leur influence dans la prise en charge de l’UB. Il en est de même pour les réalités de la prise en charge hospitalière qui ne sont pas suffisamment analysées en Côte d'Ivoire. D’où les interrogations suivantes: Quels sont les canaux d'information et les acteurs qui contribuent à réorienter les malades des soins traditionnels vers les structures modernes de prise en charge? Quelles sont les réalités quotidiennes vécues par les malades et leurs parents pendant l'hospitalisation?

L’objectif de cette étude est donc d'identifier les canaux d'information, les acteurs qui conduisent les malades de l'UB au centre de prise en charge après un détour à la médecine africaine et analyser les contraintes sociales et économiques liées à l’hospitalisation de ceux - ci.

Pour répondre à ces interrogations, après le cadre de l'étude et les outils utilisés pour collecter les données, nous présenterons, au niveau des résultats, d'abord les caractéristiques socio - démographiques des malades rencontrés et leurs localités d'origine, ensuite les canaux d’information, les facteurs sociaux modificateurs de l’itinéraire thérapeutique, enfin les réalités de l’hospitalisation et les stratégies développées.

\section{Methodologie}

Cette étude s'est déroulée au pavillon UB de l'hôpital Ahou Catherine de Djekanou dans le district sanitaire de Toumodi. Elle s'est 
adressée à 55 malades atteints de l'ulcère de Buruli et hospitalisés au centre de prise en charge de Djekanou. Précisément, elle a porté sur 29 malades hospitalisés et 20 malades ayant été hospitalisés qui venaient pour des visites médicales après leur départ du centre de prise en charge et 6 malades en soins ambulatoires.

Nous avons utilisé la technique du choix raisonné pour la selection des malades. Les critères d'inclusion de cette population d'étude étaient les suivants: avoir eu recours à la médecine traditionnelle avant d'être hospitalisé; être sous traitements médicaux. Etre hospitalisé ou avoir été hospitaliséu centre de prise en charge. L'étude s'est adressée également aux prestataires du centre de soin composés d'un médecin, de trois aides soignants et d'un aide kinésithérapeute

L'étude a combiné l'approche quantitative et qualitative. Les données ont été collectées à partir des entretiens semi - directifs et des entretiens structurés, des observations directes et d'observation participante. Pour ce faire, nous avons utilisé des guides d'entretien, des questionnaires et eu recours à des focus group.

\section{Resultats}

\section{Caracteristiques socio - demographiques de la population d'etude}

L'âge des malades rencontrés varie entre 03 ans et 54 ans. 36 malades soit $66 \%$ des malades interrogés sont des enfants et des adolescents. L'UB touche les enfants en âge d'aller à l'école ou scolarisés. D'autres malades ont été soustraits du système scolaire par leurs parents pour des soins.

Leur niveau d'instruction est faible car 38 malades soit $69 \%$ de la population d'étude ne savent pas lire et écrire. $17 \%$ de malades ont un niveau primaire de scolarisation contre $14 \%$ de malades qui ont un niveau d'étude secondaire.

Les malades du sexe féminin représentent $52 \%$ contre ceux du sexe masculin qui constituent $48 \%$ de la population d'étude. La situation matrimoniale des malades nous renseigne qu'une proportion importante (soit 81\%) de ceux -ci sont des célibataires. La situation matrimoniale des malades est relative à l'âge des malades. Après la présentation des caractéristiques socio - démographiques des malades rencontrés au centre de Djekanou, quelles sont leurs localités d’origine?

\section{Localites d'origine des malades rencontres}

Les malades rencontrés au pavillon Buruli de l'hôpital Général AHOU Cathérine de Djekanou proviennent des districts sanitaires de Toumodi, Bouaké, Tiassalé, Oumé, Soubré et de Bongouanou. 62 \% (soit 17 malades par district sanitaire) ont contracté l’UB dans les districts sanitaires 
d’Oumé et de Toumodi. $24 \%$ soit 13 parmi eux proviennent du district sanitaire de Tiassalé. Les malades des districts sanitaires de Soubré, Bouaké, Bongouanou et Sinfra représentent successivement 3,50\% des enquêtés soit 02 malades par district sanitaire.

Les distances parcourues par les malades pour se rendre au centre de prise en charge de Djekanou vont de 8 kilomètres à 230 kilomètres. Les distances les plus proches du lieu de résidence au centre de prise en charge se retrouvent chez les malades qui résident dans la commune ou la sous préfecture de Djekanou.. Les malades en provenance du district sanitaire de Soubré ont parcouru de longues distances. Ces distances vont de 100 kilomètres à environ 200 kilomètres. Pourtant des centres de prise en charge de l’UB existent dans ces différents districts sanitaires. A ce jour, 353 établissements font la prise en charge des malades dans 32 Districts Sanitaires de la Côte d'Ivoire. De cette observation, la proximité des centres de prise en charge ne résout pas encore totalement la question de leur accessibilité. Avec une telle réalité, examinons les canaux d'information qui les orientent les malades vers le centre de prise en charge.

\section{Canaux d'information et recours au centre specialise}

Cette étude s'est intéressée aux canaux d’information suivants: les médias, les campagnes de sensibilisation, les centres de santé, la famille et les amis, les agents de santé communautaires susceptibles de les orienter vers le centre de prise en charge de Djekanou.

\section{Les médias et les campagnes de sensibilisation}

Il ressort de cette étude que 90\% des malades rencontrés soutiennent n’avoir jamais entendu parler de l’UB à la radio et à la télévision. Les médias n’ont pas été la source d'information chez les malades de l'U B dans leur localité d’origine sur cette maladie. Ce n’est pas la télévision et la radio qui les ont référés au centre de prise en charge. Cette situation est illustrée par les propos suivants de la responsable des parents de malades lors d'un focus group. «A vrai dire, au village, nous ne suivons pas la télévision et la radio. Ce sont nos enfants et les jeunes qui vont souvent chez ceux qui en possèdent les soirs. Mais nous les mères, nous n'y allons pas. Ils ne nous ont jamais parlé de cette maladie et de l'existence du centre de Djekanou»

$13 \%$ des malades seulement ont été touchés par des campagnes de sensibilisation sans savoir l'existence du centre de soins de Djekanou. Cependant celles - ci n’ont pas modifiées leur itinéraire thérapeutique.

\section{Les agents de santé et la famille}

34,5\% soit 19 malades ont été référés et conduits au centre de prise en charge par les infirmiers et des médecins. La mère d'un enfant malade 
témoigne: «Quand nous avons constaté que la maladie de mon fils s'aggravait, nous avons demandé au guérisseur à retourner au village. Nous avions notre idée: aller essayer l'hôpital. Nous avons été à Oumé. C'est l'infirmier que nous avons rencontré qui nous a demandé de venir à Djekanou. Il a même souligné que la prise en charge était gratuite. C'est ainsi que nous sommes venus ici.»

21\% des malades enquêtés ont été orientés au pavillon Buruli de l'hôpital Ahou Cathérine de Djekanou par des parents d'anciens malades et des anciens malades. «Nous savions qu'à l'hôpital on soigne des plaies. Mais je n'ai jamais su qu'à Djekanou, on traite spécialement ces types de plaies (UB). C'est le petit frère de mon mari qui nous a informés. Mon fils souffrait. C'est lui qui nous a dit qu'il avait été opéré d'une hernie à Djekanou et qu'il connaît un centre de traitement des grandes plaies. C'est lui qui nous a conduits ici» (Propos d'enquêté)

La proportion de référence au centre par les ASC est moins significative soit 3,50\%.

$13 \%$ de malades sont venus directement au centre de prise en charge.

\section{Facteurs explicatifs de l'abandon des soins traditionnels}

Les malades rencontrés au cours de cette étude ont eu recours à la médecine traditionnelle avant d'être hospitalisés au centre de prise en charge de Djekanou. Parmi les facteurs qui les ont conduits à abandonner ce traitement, ils évoquent l'aggravation de la maladie et l'inefficacité de la médecine à les guérir.

\section{L’aggravation de la maladie}

Les malades soutiennent avoir une confiance aux tradipraticiens. Cela se justifie par le fait qu'ils ont guéri plusieurs cas et ils leur sont recommandés par des membres de la communauté. C’est ce que soutient un parent de malade: "Chez nous au village, le thérapeute que nous avons consulté a guéri plusieurs enfants souffrant de cette maladie. Il est reconnu comme le spécialiste en matière de lutte contre ces types de grosses plaies. Certains malades quittent des localités lointaines pour venir le consulter. C'est pour cette raison que nous l'avons consulté pendant plus de deux mois avant de venir à l'hôpital.»

Toutefois il est important de noter que malgré cette confiance aux thérapeutes traditionnels, l'aggravation de la maladie, la dégradation de l'état de santé du malade constitue un facteur de recours au centre de prise en charge spécialisé et un abandon du traitement traditionnel. Le traitement traditionnel a eu un effet négatif sur certains malades. Une dame accompagnant son enfant explique: «Mon enfant perdait du poids. Il souffrait. La plaie s'élargissait. Il sentait. J'ai été obligée d'abandonner le 
traitement par les plantes. Je ne croyais plus que c'était la sorcellerie. Je voyais mon enfant en train de mourir. Je ne pouvais plus supporter cette situation. Malgré l'insistance des parents à le traiter au village, j'ai tout refusé et comme on m'avait parlé de Djekanou, j'ai décidé de venir ici. Dieu merci, mon enfant va bien. Si j'avais écouté les autres, mon fils serait mort. J'avais peur de le perdre ou même qu'il ne puisse plus faire usage de ses membres s'il guérit»

Pour la plupart des malades rencontrés, l’aggravation de l’état de santé du malade de l' U B les a conduits à recourir au centre de prise en charge de Djekanou. Le traitement par les plantes selon ces malades présente des limites même si cette médecine a contribué à guérir certains malades. La dégradation de l'état de santé du malade occasionne une peur chez les parents qui l’assistent comme souligné dans le verbatim précédent. Il s’agit de la mort et la peur de voir le malade guérir avec un handicap.

\section{L’inefficacité de la médecine traditionnelle}

Proche des populations dans la lutte contre plusieurs pathologies, la médecine africaine présente des limites dans le traitement contre l’U B. Les malades rencontrés au centre de Djekanou témoignent n’avoir pas été guéris par le traitement par les plantes.

«J'ai consulté trois tradipraticiens avant de savoir l'existence du centre de Djekanou. Tous ces traitements n'ont fait qu'aggraver mon mal. Si j'étais parti au début de la maladie à l'hôpital, je serais guéri depuis un bon moment. Je me sens mieux maintenant. Avec les séances de rééducation, j'arrive à marcher. Les soins ici ont permis de cicatriser ma plaie alors que je sentais mauvais quand j'arrivais ici. Franchement, je me suis fait du tort en traînant avec les soins traditionnels» (Malade adulte rencontré au centre)

Pour la plupart des malades, ces soins qui vont au-delà des plantes et prennent en compte certaines prescriptions des tradipraticiens ont contribué à dégrader leur état de santé. "Le guérisseur a interdit à mon fils de consommer le manioc, l'huile, l'aubergine, le poisson et la viande. Je respectais ces prescriptions. Mais de jour en jour, mon fils perdait du poids. Imaginez vous ce qu'il mangeait quand il est interdit de consommer tout ce que j'ai cité. Il était devenu faible. Heureusement que nous sommes venus à Djekanou sinon le pire serait arrivé. Je rends gloire à Dieu». (Propos d’enquêtée)

Cette situation est évoquée par un spécialiste du centre de lutte de Djekanou. «Généralement, les malades nous arrivent dans un état de délabrement avancé avec de larges ulcérations. Ils présentent des anémies liées aux prescriptions imposées par les thérapeutes traditionnels. C'est avec les soins que nous leur donnons ici que leur santé s'améliore.» 
L’hospitalisation qui améliore la santé des malades atteints comporte des réalités.

Mais en fait, quelles sont les réalités qu'ils vivent pendant leur hospitalisation?

\section{Realites de la prise en charge hospitaliere}

La prise en charge médicale et chirurgicale du malade d'UB est gratuite à l'hôpiatl AHOU Cathérine de Djekanou. Les malades reçoivent régulièrement les soins. Le centre de prise en charge impose aux malades des conditions à remplir pendant son hospitalisation.

Premièrement, la présence d' une femme ou une jeune fille est exigée auprès du malade. Cette situation s'explique par le fait que les femmes sont habituées aux tâches de ménage et peuvent être aux petits soins du malade à savoir le laver, lui faire à manger, laver ses bandes et ses habits, l'aider à se déplacer quand le malade est dans l'incapacité de le faire.

Les images suivantes illustrent les activités des accompagnantes des malades.

Figure 1: Une mère lavant les bandes sales de sa fille atteinte d’UB

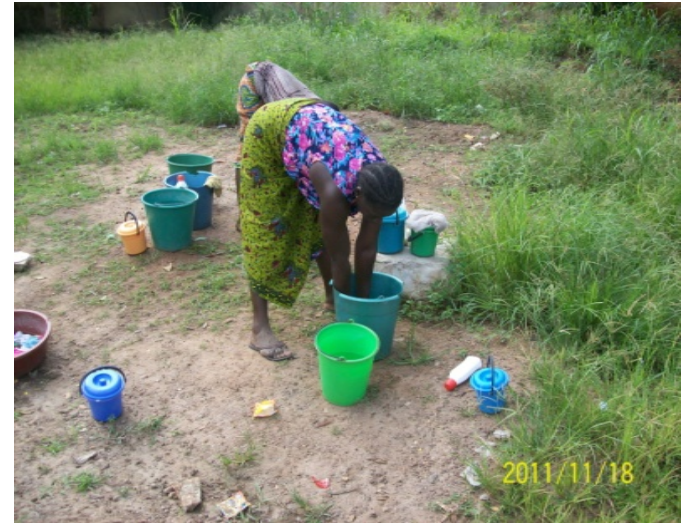

(Source: Adjet A. Abel)

Figure 2: Une mère accompagnant son fils après l'intervention chirurgicale

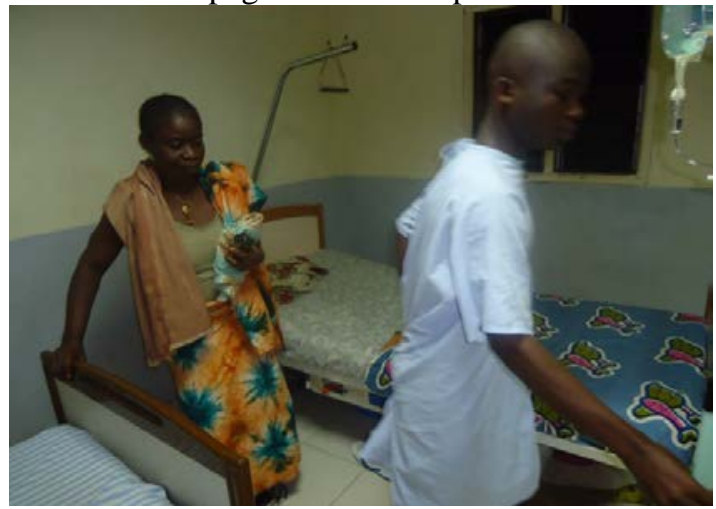

(Source: Adjet A. Abel) 
Deuxièmement, le centre de prise en charge disqualifie les femmes porteuses d'enfants parce qu'ils sont vulnérables et sont exposés aux maladies opportunistes. C'est ce que soutient un spécialiste du centre quand il affirme: "Nous interdisons que les enfants qui sont bien portants séjournent avec leurs parents au centre de prise en charge. Vous savez bien qu'ils sont vulnérables. Il faut donc les mettre à l'abri de tout danger. Il ne faut pas prendre de risque».

Troisièmement, la prise en charge impose aux malades (mères) porteuses d'enfant qui est nourri au lait maternel un sevrage. " Il n'est pas conseillé à une mère sous traitement médical d'allaiter son enfant. C'est la raison pour laquelle, nous demandons au malade dans ce cas de sevrer l'enfant et de le confier à un parent qui n'est pas au centre.» soutient un spécialiste du centre. Trois cas ont été rencontrés au cours de cette étude. Il s'agit des malades K.A.A., K. A, et N. A.

L'hospitalisation des malades de l'UB est marquée par de diverses difficultés. Les rapports entre les malades, leur parent qui les accompagne et le centre de prise en charge apparaissent souvent conflictuels au regard des règlements qu'il impose aux malades.

La séparation entre la mère et son enfant devient plus difficile quand l'enfant dont la garde a été confiée à sa grande mère au village décède. C'est le cas de K. A. A. Son fils qui a été sevré et confié à sa grand-mère pendant son hospitalisation est décédé au village.

Quatrièmement, la durée de l'hospitalisation allant de trois à sept mois pour les malades rencontrés et les difficultés financières constituent un ensemble d'inquiétudes auxquelles les malades font face. Le réfectoire des malades construit par l'aide de la coopération japonaise au développement au sein de l'hôpital général de Djekanou n'est pas fonctionnel. L'hospitalisation épuise financièrement les parents des malades qui leur apportent une assistance. "Nous n'avons plus d'argent. C'est difficile de nous faire à manger souvent. Au village, ce qui nous viennent en aide aussi n'ont plus d'argent.» Propos recueillis d'un focus group.

Les malades et leurs parents ont régulièrement recours à leurs familles au village pour de l'assistance financière et des vivres (ignames, bananes, riz, ingrédients pour la sauce). Souvent, la demande ne connaît pas des échos favorables ou reçoit une suite favorable tardivement. "Nous rencontrons des difficultés ici. Nous n'avons plus d'argent pour nous occuper des malades. Quand on envoie des messages au village, ce n'est pas évident. Il n'y a rien aussi là - bas. Difficile de se trouver de quoi pour se nourrir des fois ici » (Focus group) C'est ainsi qu'ils développent des stratégies perceptives en terme de résilience pour faire face aux difficultés liées à la prise en charge hospitalière. 


\section{Strategies developpees pendant l'hospitalisation}

Les coûts indirects liés à l'hospitalisation des malades de l'UB sont à la charge du malade et de sa famille. C'est pour remédier à cette difficulté qu’ils développent des résiliences pour faire face à certaines difficultés liées à la prise en charge.

Ainsi, les parents de malades présents au centre de prise en charge deviennent - ils une potentielle main d'œuvre pour les habitants de la ville et des villages environnants sous deux formes d'organisation mises en place par les femmes qui assistent les malades.

Elles décident d'accompagner volontairement des villageois dans leurs champs. En compensation, elles reviennent avec des ignames, des bananes, du manioc ou du fagot pour pouvoir faire face aux charges de nourriture.

Il y a aussi le «contrat» ou le «téré kélé » en malinké qui consiste à faire une journée de travaux champêtres. En retour, chaque participante reçoit 1000 F CFA pour sa prestation journalière. Les ressources financières obtenues du «téré kélé bara» leur permettent d'acheter des morceaux de savon, du poisson et faire à manger pour elles et les malades.

D’autres accompagnants de malades s'adonnent à des activités de triage des grains de café dans les moulins à moudre le café de la ville contre de l'argent. Elles collectent également des boîtes vides, des pots, des bouteilles dans des poubelles de la ville et aux alentours de l'hôpital qu'elles lavent au centre pour revendre.

Certains parents de malades se regroupent par affinité (soit un groupe de 2 ou 3 personnes), se cotisent et font ensemble la nourriture. Ainsi les malades et les parents du groupe partagent- ils le repas ensemble. Au sein du centre de prise en charge se développe entre les parents de malades la solidarité.

\section{Discussions}

Cette étude nous a permis d'identifier les facteurs qui orientent les malades d'UB vers le centre de prise en charge et les contraintes sociales et économiques liées à leur hospitalisation.

Les résultats de cette étude rejoignent celle de Johnson et al (2004). Dans un contexte diversifié de services de soins, les patients atteints d'UB utilisent selon leur croyance, leur perception de la maladie et leur possibilité financière l'un ou l'autre des niveaux de soins. Ce n'est qu'après l'échec de l'automédication par plantes ou par produits achetés sur le marché qu’ils décident de recourir soit au centre de santé.

Quant aux frais, l'hospitalisation des malades de l'U B comporte des frais indirects malgré la gratuité des soins médicaux et chirurgicaux. Cette situation est décrite par ( Asiedu, 2000) quand il affirme que les frais 
indirects comprennent le prix de la nourriture et l'équivalent de la perte de gain du patient et de son garde - malade.

Par ailleurs, le centre de prise en charge de Djekanou n’offre pas gratuitement la nourriture aux malades. De ce fait, l'hospitalisation est coûteuse pour les malades. Cette situation est souvent différente chez le tradithérapeute à qui le malade recourt avant son hospitalisation. Au Benin, c'est la famille du tradithérapeute qui nourrit le patient. En termes de coût indirect et de disponibilité pour les activités économiques, le traitement traditionnel constitue un avantage pour le patient (Johnson et al, 2004).

Pour l'OMS (2009), même si les coûts ne sont pas clairement énoncés, il en résulte que chaque service est payant au final. Le traitement traditionnel peut être tout aussi cher, si non pas plus onéreux que le traitement moderne.

La sensibilisation n’a pas été l'élément de motivation au recours à l'hospitalisation chez les malades rencontrés à Djekanou. Contrairement à cette réalité, Debacker et Coll (2004) ont montré que la sensibilisation dans les villages ainsi qu'un bon traitement contribuent à diminuer la médiane d’hospitalisation qui peut passer de 267 jours en 1997 à 30 jours en 2001.

Handicap International - Togo (2013) dans une étude réalisée au Togo démontre que 91,4 \% des personnes interrogées ont eu l’opportunité au moins une fois d'assister à une campagne de sensibilisation au sein de leur communauté à la différence de cette étude où $13 \%$ des malades ont été touchés par une campagne de sensibilisation.

\section{Conclusion}

Au terme de cette étude, il est important de noter que les Agents de Santé Communautaires (ASC), les campagnes de sensibilisation, la radio et la télévision n’ont pas véritablement contribué à orienter les malades vers le centre de prise en charge de Djekanou. Ces canaux d'informations n'ont pas joué rôle important dans le changement de l'itinéraire thérapeutique des malades. Ce sont les infirmiers et médecins et les parents de malades qui ont référé et conseillé à près de la moitié des malades de recourir au centre. Toutefois, l'aggravation de la maladie et l'inefficacité des soins traditionnels ont conduit les malades à accepter l'hospitalisation. La prise en charge hospitalière de l'UB est marquée par des difficultés d'ordre social, psychologique et économique. Il convient de sensibiliser les populations à un recours au stade précoce de la maladie afin d’éviter les longues périodes d’hospitalisation et les contraintes liées à celle -ci.

\section{Remerciements.}

Nous tenons à remercier toutes les personnes et institutions qui ont contribué à la réalisation de cette étude. Au personnel de l’hôpital Général 
Ahou Cathérine de Djekanou Aux malades et leurs parents que nous avons rencontrés Au P.N.L.U.B et à son Directeur coordonnateur Professeur Henri ASSE Au consortium de recherche AFRIQUE ONE. Cette étude a été préparée avec le soutien du consortium Afrique One «Ecosystème et santé de la population: Elargir les frontières de la santé» Afrique One est financé par le Wellcome Trust (WT087535MA).

\section{References:}

1. Adjet, A., Adou, D., Daniele, O., 2016, Itinéraires thérapeutiques pluriels et recours tardif des malades de l'ulcère de Buruli dans les centres de prise en charge dans le district sanitaire de Yamoussoukro (Côte d'Ivoire), ESJ, vol 12, Nº 30

2. Asiedu, K., scherpbier, R. et Raviglione, M. (2000). Ulcère de Buruli Infection

Mycobacterium ulcerans. Genève : OMS.

3. Aujoulat I, Ribs M-P et Koita Y, 1996, «l'ulcère de Buruli: un problème de santé publique méconnu, appelant une mobilisation internationale » in Développement et Santé, n¹25, Octobre

4. Handicap I, 2013, Enquête CAP sur l'ulcère de Buruli au sein de la population de Zio et Yoto

5. Johnson R. C, Makoutode M, Hougnihin R, et al (2004). Le traitement traditionnel de l'ulcère de Buruli au Bénin. Med Trop; 64 : $145-50$

6. Kibadi K., 2004, Enquête Connaissances - attitudes - pratiques (CAP) de la population Songololo (RD Congo) sur l'ulcère de Buruli. Bull Soc Pathol Exot (97) 4

7. Kibadi K., 2007, «Etude des appellations et des représentations attachées à l'infection à mycobacterium ulcerans dans différents pays endémiques d'Afrique», Med Trop.

8. Laterali M., 2005, Ethnographie de la constitution d'un problème de santé publique au Cameroun : l'exemple de l'ulcère de Buruli ou atom dans l'arrondissement d'Ayos, Mémoire de licence en ethnologie, Université de Neuchâtel.

9. ORGANISATION MONDIALE DE LA SANTE., 2001, Ulcère de Buruli. Prise en charge de l'infection à Mycobacterium ulcerans, WHO/CDS/CPE/GBUI/.3, $72 \mathrm{p}$

10. ORGANISATION MONDIALE DE LA SANTE, 2009, Réunion annuelle de l'initiative mondiale contre l'ulcère de Buruli, 31 Mars 02 Avril. Résumé. Cotonou: OMS

11. PNLUB, 2009, Le bulletin de l'ulcère de Buruli, octobre- novembredécembre 
12. SENE E, 2013, «Ulcère de Buruli: Prise en compte de l'expérience de la maladie pour une prise en charge culturellement compétente» Travail de Bachelor dans le cadre de la formation HES, Genève 